\title{
ON INDUCED PROJECTIVE INDECOMPOSABLE MODULES
}

\author{
PETER SIN AND WOLFGANG WILLEMS
}

(Communicated by Bhama Srinivasan)

\begin{abstract}
A well-known theorem of Fong states that over large enough fields of any characteristic, the principal indecomposable modules of a soluble finite group are induced from subgroups of order prime to the characteristic. It is shown that this property in fact characterises soluble finite groups.
\end{abstract}

\section{INTRODUCTION AND STATEMENT OF THE MAIN THEOREM}

Fong [7] has shown that over a large enough field of characteristic $p$, all the projective indecomposable modules of a $p$-soluble finite group are induced from a $p$-complement. Let $G$ be a finite group. If one postulates the existence of a $p^{\prime}$-subgroup $H$ from which all the projective indecomposable modules are virtually induced, then it is not hard to show, by considering dimensions of projective modules, that $H$ must be a $p$-complement. However, $G$ need not be $p$-soluble, as illustrated by the example below. Nevertheless, if one makes the same assumption for all primes, then $G$ is soluble by P. Hall's theorem. We consider a weaker hypothesis, in which the $p^{\prime}$-subgroups are allowed to depend on the projective module to be induced, which in some ways resembles the definition of an $M$-group. We now state our result; we shall assume all fields to be splitting fields for all groups which occur.

Theorem. Let $G$ be a finite group. If for every prime $p$, all the projective indecomposable modules for $G$ in characteristic $p$ are induced from $p^{\prime}$-subgroups, then $G$ is soluble.

To see that a $p$-local version of the theorem does not hold, take $G$ to be the alternating group $A_{5}$ and $p=5$. It is not hard to check that the three 5-modular irreducible modules of dimensions 1,3 and 5 have projective covers induced from subgroups isomorphic to $A_{4}, S_{3}$, and $A_{4}$ respectively. Moreover, all projective modules are virtually induced from $A_{4}$.

In the same vein, we mention the Fong-Swan-Isaacs theorem that each irreducible $p$-modular character of a $p$-soluble group is liftable to a $p$-rational

Received by the editors December 18, 1987 and, in revised form, April 1988.

1980 Mathematics Subject Classification (1985 Revision). Primary 20C20.

The first author was supported by NSF grant DMS 8421367 .

The second author was supported by the DFG and the NSF. 
complex character. G. Hiss has proved that a finite group satisfying the conclusion of this theorem for all primes must be soluble [8]. Again $A_{5}$ provides an example against a $p$-local version.

\section{Preliminaries}

Lemma 2.1. Let $B$ and $C$ be subgroups of $A$, with $B \unlhd A$. If the $k A$-module $V$ is induced from $C$ then the $k(A / B)$-module $V \otimes_{k A} k(A / B)$ is induced from $B C / B$.

Proof. We have a natural isomorphism

$$
\left(W \otimes_{k C} k A\right) \otimes_{k A} k(A / B) \cong\left(W \otimes_{k C} k(B C / B)\right) \otimes_{k(B C / B)} k(A / B),
$$

for any $k C$-module $W$, obtained by factoring the natural map $C \rightarrow A / B$ in two ways (associativity of tensor product).

Lemma 2.2. Let $B \unlhd A$ and let $V$ be an irreducible $k(A / B)$-module. Let $P_{A}$ and $P_{A / B}$ denote the $k A$ - and $k(A / B)$-projective covers of $V$. Then

$$
P_{A / B} \cong P_{A} \otimes_{k A} k(A / B) \text {. }
$$

Proof. The right-hand side is clearly a projective $k(A / B)$-module with $V$ as its head.

Lemma 2.3. Let $B \unlhd A$, and suppose that $V$ is an irreducible $k A$-module such that $V_{B}$ is a multiple of some irreducible $k B$-module $W$. If some multiple of the projective cover $P(V)$ of $V$ is induced from a subgroup $C$ then a (possibly different) multiple of $P(W)$ is induced from $B \cap C$. In the special case where $V=k$ is the trivial module, its $k B$-projective cover is a transitive permutation module if its $k A$-projective cover is.

Proof. Since $V_{B}$ is a multiple of $W$, it follows from [6, II, 2.11] that $P(V)_{B}$ is a multiple of $P(W)$. On the other hand, applying Mackey's formula to our hypothesis yields

$$
P(V)_{B}=X_{C}{ }_{B} \cong \bigoplus(X \otimes g)_{C^{8} \cap B}{ }^{B},
$$

for certain elements $g \in G$. In particular, $X_{C \cap B}{ }^{B}$ is a multiple of $P(W)$. If $V=k$, then we have

$$
k_{C \cap B}{ }^{B} \cong e P\left(k_{N}\right)
$$

and Frobenius reciprocity implies $e=1$.

2.4. A reduction step. Let $H$ be a minimal counterexample to the theorem of $\S 1$. If $N$ is a minimal normal subgroup then by Lemmas 2.1 and $2.2 H / N$ is soluble. Therefore $N$ must be the direct product of copies of some nonabelian simple group $G$. The theorem will be derived from the classification of finite simple groups.

\section{GROUPS OF LIE TYPE}

In this section we shall always assume that the simple subnormal subgroup $G$ of the minimal counterexample $H$ is of Lie type, of characteristic $p$, say. 
The main idea used to eliminate these groups is a reduction of the problem to groups of small rank by means of the Steinberg module. Explicit information about the groups of low rank is then applied.

Lemma 3.1. Suppose $G$ has a split BN-pair of characteristic $p$. Then

(a) A multiple of the Steinberg module $S t_{G}$ is induced from a $p^{\prime}$-subgroup of $G$.

(b) Let $G_{J}$ be a parabolic subgroup of $G$ with Levi factor $L_{J}$. Then some multiple of $S t_{L_{J}}$ is induced from a $p^{\prime}$-subgroup of $L_{J}$.

Proof. (a) Let $N \cong G \times \cdots \times G$ be a minimal normal subgroup of $H$ and choose an irreducible $k H$-module lying over the irreducible $k N$-module $W:=$ $S t_{G} \otimes \cdots \otimes S t_{G}$. Since $W$ is $H$-stable, Lemma 2.3 implies that some multiple of $P(W)$ is induced from a $p^{\prime}$-subgroup of $N$. The assertion follows from a further application of Lemma 2.3 with $G$ as a normal subgroup of $N$.

(b) The module $S t_{L_{J}}$ may be regarded as a $k G_{J^{-}}$-module. Let $P$ be its $k G_{J^{-}}$ projective cover. It is well known that $P \cong\left(S t_{G}\right)_{G_{J}}$ (see [3, 6.3.3]). By (a) some multiple of $S t_{G}$ is induced from a $p^{\prime}$-subgroup of $G$. It follows from Mackey decomposition that some multiple of $P=\left(S t_{G}\right)_{G_{J}}$ is induced from a $p^{\prime}$-subgroup of $G_{J}$. Lemmas 2.1 and 2.2 now give the result.

3.2. A reduction step. Suppose that $G$ is not the Tits group ${ }^{2} F_{4}(2)^{\prime}$. Then $G$ has a split $B N$-pair in the defining characteristic, so Lemma 3.1 applies. Thus for any Levi factor $L_{J}$, a multiple of the Steinberg module is induced from a $p^{\prime}$-subgroup of $L_{J}$. Since $Z\left(L_{J}\right) \leq \operatorname{Ker} S t_{L_{J}}$, the same is true of the quotient $L_{J} / Z\left(L_{J}\right)$, by Lemma 2.1. If $J$ is connected and $L_{J}$ is insoluble and not of type $B_{2}(2)$, then the derived subgroup $L$ of $L_{J} / Z\left(L_{J}\right)$ is a simple group of Lie type in the same characteristic as $L_{J}$. In this case a multiple of $S t_{L}$ is induced from a $p^{\prime}$-subgroup, by Lemma 2.3, since the Steinberg module of $L_{J}$ remains irreducible upon restriction to $L$. Thus a major part of the elimination of the simple groups of Lie type will be accomplished by proving, for various simple groups $L$ of low rank, the impossibility of the equation

$$
e S t_{L} \cong X_{U}{ }^{L} \text {, }
$$

where $X$ is an irreducible module for a $p^{\prime}$-subgroup $U$ of $L$ and $e$ is a natural number.

We shall use the following result repeatedly.

Lemma 3.3. Let $L$ be a group of Lie type and $U$ a $p^{\prime}$-subgroup such that $(*)$ holds. Then $U$ contains a conjugate of every cyclic $p^{\prime}$-subgroup of $L$.

Proof. Let $\chi_{S t}$ be the complex character of a lift of $S t_{L}$. The lemma follows from the formula for induced characters and the fact that $\chi_{S t}$ does not vanish at $p^{\prime}$-elements $[3,6.4 .7]$. 
In the next step we eliminate all simple groups $G=L(q)$ of Lie type, in which the field parameter $q$ is greater than 7. By 3.2, it is enough to show that (*) never holds for simple rank 1 groups $L$ with $q>7$.

3.4. Groups of rank $1, q>7 .^{1}$ In each of the following cases we assume that (*) holds and obtain a contradiction.

(a) $L={ }^{2} B_{2}(q), q=2^{2 s+1}, s \geq 1$ : All Sylow subgroups of odd order are cyclic, so by lemma 3.3, $L$ has a 2-complement. But none exists (see [12]).

(b) $L=\operatorname{PSL}(2, q), q=p^{s}>7$ if $p$ is odd, and $q \geq 4$ if $p=2$ : If $p=2$, then all Sylow subgroups of odd order are cyclic, giving rise by Lemma 3.3 to a nonexistent 2-complement. If $p$ is odd, then all Sylow subgroups for primes other than $p$ are cyclic or dihedral, so that the subgroup $U$ of $(*)$ must have order at least $\frac{1}{4}(q+1)(q-1)$. Dickson's list of subgroups of $\operatorname{PSL}(2, q)$ and the assumption that $q>7$ leave only the possibility that $L=\operatorname{PSL}(2,11)$ and $U \cong A_{5}$. By comparing dimensions and Frobenius reciprocity, we have $e=\operatorname{dim} X=\left(\left(S t_{L}\right)_{U}, X\right)$, which is incompatible with the character degrees of $A_{5}$ and the fact that $S t_{L}$ is 11-dimensional.

(c) $L={ }^{2} G_{2}(q), q=3^{2 s+1}, s \geq 1:$ It is known [9, XI, 13.2] that the Sylow $r$-subgroups are cyclic for $r \neq 2,3$. Thus by Lemma 3.3 we have

$$
|L|_{\{2,3\}^{\prime}}|| U|||L|_{3^{\prime}} \text {. }
$$

Also, the Sylow 2-subgroups are known to be elementary abelian or order 8 [9, XI, 13.2]. Since the Suzuki groups of a) are the only nonabelian simple groups of order not divisible by 3, and these have larger 2-parts than $L, U$ must be soluble, and we may deduce the following structure for it.

$$
\begin{array}{cc}
U=G_{\delta 2^{\prime}, 2,2^{\prime}}(U) & \bullet \\
G_{\delta 2,2^{\prime}}(U) & \stackrel{\bullet}{\mid} \leq \leq Z_{7} \\
K=G_{\delta 2^{\prime}}(U) & \dot{\mid} \leq Z_{2} \times Z_{2} \times Z_{2} \\
F(K) & \dot{\mid\}} \\
1 & \dot{\mid}
\end{array}
$$

Here $F(K)$ denotes the Fitting subgroup of $K$. Now $L$ has cyclic Hall subgroups $M^{+}$and $M^{-}$of orders $q+3 m+1$ and $q-3 m+1$ respectively, where $m=3^{s}[9, \mathrm{XI}, 13.2(\mathrm{f})$ and $(\mathrm{g})]$. We may assume these are contained in $U$.

\footnotetext{
${ }^{1}$ The referee has suggested the following argument which would cover the cases in which $q>11$ is a power of a prime $p>3$ : Choose a cyclic maximal torus $T$ as given in Rational groups and related topics, $W$. Feit, G. Seitz. By 3.3, we may assume $T \leq U$. Now for the values of $q$ considered, one knows from (10.2) of The root subgroups of maximal tori in finite groups of Lie type, G. Seitz, Pacific J. Math. 106 (1983), 153-244, the structure of $U$. In particular, $T \unlhd U$ as $p+|U|$. This gives a contradiction as one can find some prime $l$ other than $p$ which does not divide $|N(T)|$, since by $3.3, l$ divides $|U|$.
} 
Let $M$ be either $M^{+}$or $M^{-}$. Then for $x \in M$ it is known that $C_{L}(x)=M$ [9, XI, 13.2(f)]. Clearly, $F(K)$ is nontrivial so we may choose a prime $r$ dividing its order. If $r|| M \mid$, then $M \unlhd U$, which contradicts the fact that

$$
\left|N_{U}(M): M\right| \mid 2 \text {. }
$$

Suppose then, that $\left(|F(K)|,\left|M^{+}\right|\left|M^{-}\right|\right)=1$. It follows from the structure of $L$ that $F(K)$ is cyclic of order dividing $\frac{1}{8}(q+1)(q-1)$. Pick a nontrivial subgroup $J$ of $F(K)$ of prime order. Then both $M^{+}$and $M^{-}$act faithfully on $J$ so we have

$$
q^{2}-q+1=\left|M^{+}\right|\left|M^{-}\right||| \operatorname{Aut}(J) \mid \leq \frac{1}{2}(q-1),
$$

a contradiction.

(d) Finally we have to show that for $L=\operatorname{PSU}\left(3, q^{2}\right), q^{2}=p^{2 s}>4$, the equation $(*)$ leads to a contradiction. The order of $L$ is

$$
\frac{1}{d} q^{3}\left(q^{2}-q+1\right)\left(q^{2}-1\right)(q+1)
$$

where $d=(3, q+1)$. Pick a Zsigmondy prime $r \mid q^{6}-1=p^{6 s}-1$ with $r \nmid p^{j}-1$ for $1 \leq j<6 s$. Thus $r \nmid q^{3}-1$ and $r \nmid q^{2}-1$ so $r \mid q^{2}-q+1$. Since $p^{r-1} \equiv 1(r)$, we have $r \geq 7$. According to [10] we know the structure of the Sylow subgroups of $L$. For instance, the Singer cycle of order $\left(q^{2}-q+1\right) / d$ is a Hall subgroup and the normaliser of a Sylow $p$-subgroup has a cyclic complement of order $\left(q^{2}-1\right) / d$. Thus, Lemma 3.3 and $(*)$ imply

$$
\frac{1}{d}\left(q^{2}-q+1\right)\left(q^{2}-1\right)|| U \mid .
$$

Suppose first that the generalised Fitting subgroup $F^{*}(U)$ is soluble. Let $R$ be a subgroup of order $r$ and choose a Singer cycle $S$ with $R \leq S \leq U$. If $R \leq F^{*}(U)$ then $R \unlhd U$ since the Sylow $r$-subgroups are cyclic. But $S \cap S^{y}=1$ unless $y \in N_{L}(S)$ so that $N_{L}(S)=N_{L}(R)$, contradicting (**), since $\left|N_{L}(S)\right|=3|S|$. Therefore we may assume that $R \nsubseteq F^{*}(U)$. Since $C_{U}\left(F^{*}(U)\right) \leq F^{*}(U)$, we may find a Sylow subgroúp $T \leq F^{*}(U)$ which is not centralised by $R$. As the ranks of all Sylow subgroups of $L$ are bounded by 2 , and $r \geq 7$, the order of $T$ is not a power of 2 or 3 . This means in particular that $T$ is abelian, so may be decomposed as $T=C_{T}(R) \times[T, R]$. Then $E=[T, R] R$ is a Frobenius group. Since neither $p$ nor 3 divides the order of $E$, we may lift its 3-dimensional modular, projective representation as a subgroup of $L$ to a faithful 3-dimensional ordinary complex representation. This is contrary to the fact that a faithful character of a Frobenius group must have degree no less than the order of the Frobenius complement.

It remains to tackle the case that $F^{*}(U)$ has an insoluble composition factor. Let $\widetilde{U}$ be the inverse image of $U$ in $\operatorname{SU}\left(3, q^{2}\right)$. From the 2-structure of $\operatorname{SU}\left(3, q^{2}\right)$ it follows that the layer $\tilde{Y}$ of $F^{*}(\tilde{U})$ is a quasisimple group. Since 
$p \nmid|\widetilde{Y}|$, we may lift the representation of $\widetilde{Y}$ to obtain a faithful 3-dimensional complex representation. Now $\bar{Y}=\widetilde{Y} / Z(\widetilde{Y})$ cannot be a sporadic group, since none has a nontrivial projective representation of degree 3 (see [5]). If $\bar{Y}$ is an alternating group then from the 2-rank, the only possibilities are $A_{5}, A_{6}$ and $A_{7}$. Now $A_{7}$ has no nontrivial projective representations of degree 3 (see [5]), and the two other groups are of Lie type. We are therefore reduced to the case that $\bar{Y}$ is of Lie type, and has a nontrivial 3-dimensional projective representation. By a result of Landazuri and Seitz [11], this can only happen if $\bar{Y}$ is one of $\operatorname{PSL}(2,5), \operatorname{PSL}(2,7)$ or $\operatorname{PSL}(2,9)$. Choose an element $x$ of order $r$ in the inverse image of $R$ in $\tilde{U}$. Then $x$ acts nontrivially on $\tilde{Y}$ since $S$ has the trivial intersection property. As $r \geq 7$ and $|Z(\widetilde{Y})| \mid 6, x$ acts trivially on $Z(\tilde{Y})$. We argue that $\bar{Y} \cong \operatorname{PSL}(2,7)$ and $r=7$; otherwise, $x$ would act trivially on $\bar{Y}$, and for $y \in \tilde{Y}$ we would have $y^{x}=y z$ for some $z \in Z(\tilde{Y})$, and then $y^{x^{6}}=y z^{6}=y$, contradicting the order of $x$. Therefore $\bar{Y} \cong \operatorname{PSL}(2,7)$ and $r=7$, and it follows that $q^{2}-q+1|| \operatorname{PSL}(2,7) \mid .2=2^{4} .3 .7$, whence $q=5$. Thus by $(* *), \operatorname{PSU}\left(3.5^{2}\right)$ has a $5^{\prime}$-subgroup of order divisible by $2^{3}$.7.3. Since the subgroup of order $q^{2}-1=8$ is cyclic, and $U$ is insoluble, we even have a subgroup of order divisible by twice this number. But such a subgroup does not exist (see [5]).

3.5. Groups of low rank, $q \leq 7$. Up to this point, we have proved that in a minimal counterexample the simple group $G=L(q)$ satisfies $q \leq 7$. Since it happens over small fields that the Steinberg module for groups of low rank may satisfy $(*)$ (for instance if the group is soluble), for these fields we must look to groups of higher rank to provide the base of our induction. Explicitly, we have two lists:

(i) $\operatorname{PSL}(2,4), \operatorname{PSL}(3, q)$ for $q=3,4,5$ and $7, \operatorname{PSL}(4,2), \operatorname{PSU}(4,4)$, $\operatorname{PSU}(5,4)$.

(ii) $\operatorname{PSL}(2,7), \operatorname{PSL}(3,2), B_{2}(2)^{\prime}, G_{2}(2)^{\prime}, G_{2}(q)$ for $q=3,4,5$ and 7, $\operatorname{PSp}(4, q)$ for $q=3,4,5$ and $7, \operatorname{PSp}(6,2), F_{4}(2)^{\prime}$.

If $L(q)$ is any simple group of Lie type with $q \leq 7$, then either it belongs to the second list or else it has a parabolic subgroup with a composition factor in the first list. The impossibility of $(*)$ for the groups in list (i) is easily verified using Lemma 3.3 and the lists of maximal subgroups in the ATLAS [5].

As for the groups in (ii), the groups $G_{2}(2)^{\prime} \cong \operatorname{PSU}(3,9)$ and $B_{2}(2)^{\prime} \cong$ $\operatorname{PSL}(2,9)$ are covered by the case $q>7$. Most of the others can be dealt with using the last part of Lemma 2.3. This tells us that for the simple group $G$, the projective cover of the trivial module in every characteristic is a transitive permutation module. Where possible we choose a prime which divides the group order but whose square does not, and find candidates for the projective cover $P(1)$ of the trivial module from the ATLAS character tables, the choice being severely restricted by general properties of projective characters and Brauer trees (see the discussion in $\S 4)$. We give a list of pairs $(G, r)$ of the groups and 
primes.

$$
\begin{gathered}
(\operatorname{PSL}(2,7) \cong \operatorname{PSL}(3,2), 3),\left(G_{2}(3), 7\right),\left(G_{2}(4), 7\right),\left(G_{2}(5), 7\right), \\
(\operatorname{PSp}(4,3), 5),(\operatorname{PSp}(4,4), 17),(\operatorname{PSp}(4,5), 13),(\operatorname{PSp}(6,2), 5),\left(F_{4}(2)^{\prime}, 13\right) .
\end{gathered}
$$

None of the possible degrees divides the group order, except in the case of $\operatorname{PSp}(4,4)$. Here the only possibility would give a permutation character of norm 2 and degree 51, but it is known (see [2]) that this group has no doubly transitive permutation representations. Using the known degrees of the principal series characters for $G_{2}(7)$ [4], the dimension of $P(1)$ in characteristic 43 is found to be $4257=43 \times 99$, which does not divide the group order. Similarly, using the degrees of the principal series characters of $\operatorname{PSp}(4,7)$ (see $[3$, p. 448]), we find that in characteristic 5, $P(1)$ gives a complex character of norm 2 and degree 225. On the other hand we know (from [2], for example) that this group has no doubly transitive permutation representation.

In view of $3.1,3.2,3.4$ and 3.5 , we have finished proving that the simple group $G$ in a minimal counterexample is not of Lie type.

\section{AlternATING GROUPS AND SPORADIC SIMPLE GROUPS}

We return to a minimal counterexample $H$ to the theorem. In the previous section, the groups of Lie type were eliminated as candidates for the simple subnormal subgroup $G$ of $H$. We now consider the possibilities that the group $G$ is an alternating group $A_{n}$ or a sporadic group. In the former case, we know also that $n \geq 7$ and $n \neq 8$, since $A_{5} \cong \operatorname{PSL}(2,5), A_{6} \cong \operatorname{PSL}(2,9)$ and $A_{8} \cong \operatorname{PSL}(4,2)$. From Lemma 2.3 we have that for every prime $p$, the $p$ projective cover, $P(1)$, of the trivial module is a transitive permutation module. We assume these conditions for the rest of this section. We first point out the relevance of Brauer trees to the present situation.

Lemma 4.1. If the principal p-block has cyclic defect groups and the node in the Brauer tree adjacent to the trivial node is not exceptional, then $P(1)$ is a doubly transitive permutation module.

Lemma 4.2. Suppose $n \geq 7$ and $p$ is a prime with $n / 2<p \leq n$. Then the principal $p$-block of $A_{n}$ has cyclic defect groups and the node adjacent to the trivial module is not exceptional.

Proof. Since $p$ is odd, $P(1)$ is the restriction to $A_{n}$ of the projective cover $Q(1)$ of the trivial module for $S_{n}$. The principal $p$-block of $S_{n}$ is also of defect one, and its Brauer tree has no exceptional node, since all the characters of $S_{n}$ are rational. Let $\Phi$ be the character of $Q(1)$. Then $\Phi=1+\chi$, for some irreducible character $\chi$. We must show that $\chi_{A_{n}}$ is irreducible. We may assume that $\chi(1)>1$. From the Brauer tree for $S_{n}$ we see that the restriction of $\chi$ to the $p$-regular elements is $1+\beta$, for some irreducible Brauer character $\beta$. Suppose that $\chi_{A_{n}}=\theta+\theta^{\sigma}$. Then the trivial Brauer character must be a constituent of the restriction to the $p$-regular elements of one, hence both of $\theta$ 
and $\theta^{\sigma}$. Therefore $\beta_{A_{n}}$ has the trivial Brauer character as a constituent and so is a multiple of it. Thus all $p$-regular elements lie in the kernel of $\chi=1+\beta$, so since this kernel is nontrivial, it must be $A_{n}$, and $\chi$ must be the sign character, contradicting the assumption that $\chi(1)>1$.

Lemma 4.3. The simple group $G$ in the minimal counterexample is not an alternating group.

Proof. It suffices to eliminate the groups $A_{n}, n \geq 6, n \neq 8$. By Bertrand's postulate, we may choose a prime $p$ with $n / 2<p<n$. Then by Lemmas 4.1 and 4.2, $P(1)$ is a doubly transitive permutation module. These were determined by Maillet (see [2]). Apart from the natural representation of degree $n$, only $A_{7}$ and $A_{8}$ have doubly transitive permutation representations, each having one of degree 15 . Since $p$ does not divide $n$, the natural permutation module is not projective. Therefore it remains to dispose of $A_{7}$. However, as there is a subgroup of index 15 our strategy fails in this case. Fortunately, the dimension of $P(1)$ in characteristic 2 is known to be 72 (see [1]), and a subgroup of order 35 would have to be cyclic, which is absurd. This completes the proof of the lemma.

Our final result shows that the theorem is a consequence of the classification of the finite simple groups.

Lemma 4.4. In the minimal counterexample $H$, the simple group $G$ is not one of the 26 sporadic groups.

Proof. In each case, we find a prime $p$ which divides the group order, but whose square does not. Then we find from the ATLAS character tables a list of candidates for the character (or characters) adjacent to the trivial node of the Brauer tree for the principal $p$-block. The following elementary facts reduce the number of possibilities:

(a) If only one irreducible character is next to the trivial node, then it must be rational and take the value -1 on $p$-elements.

(b) If the node next to the trivial node is exceptional, then all its characters have the same degree, and the sum of their values on a class of $p$ elements must be -1 .

From this list, we obtain a shortlist of possible dimensions of $P(1)$. In most cases, the numbers on this shortlist do not divide the group order. In the five cases which remain, we are able to inspect the lists of maximal subgroups; in the ATLAS to see that there is no subgroup of the given index. This proves the lemma. The pairs $(G, p)$ consisting of a sporadic group and the prime chosen are given below; an asterisk indicates that the list of maximal subgroups was 
used to obtain the contradiction.

$$
\begin{gathered}
\left(M_{11}, 5\right) *,\left(M_{12}, 11\right) *,\left(J_{1}, 19\right),\left(M_{22}, 7\right),\left(J_{2}, 7\right), \\
\left(M_{23}, 7\right) *,(H S, 7),\left(J_{3}, 19\right) *,\left(M_{24}, 11\right) *,(M c L, 11), \\
(H e, 17),(R u, 7),(S u z, 7),\left(O^{\prime} N, 5\right),\left(C o_{3}, 7\right),\left(C o_{2}, 11\right), \\
\left(F i_{22}, 13\right),(H N, 7),(L y, 7),(T h, 13),\left(F i_{23}, 17\right), \\
\left(C o_{1}, 23\right),\left(J_{4}, 5\right),\left(F i_{24}^{\prime}, 29\right),(B, 31),(M, 17) .
\end{gathered}
$$

\section{REFERENCES}

1. D. Benson, Modular representation theory, new trends and methods, Lecture Notes in Math., vol. 1081, Springer-Verlag, Berlin and New York, 1984.

2. P. Cameron, Finite permutation groups and finite simple groups, Bull. London Math. Soc. 13 (1981), 1-22.

3. R. W. Carter, Finite groups of Lie type, conjugacy classes and complex characters, Wiley, Chichester, 1985.

4. B. Chang and R. Ree, The characters of $G_{2}(q)$, Symposia Math. 13 (1974), 395-413.

5. J. H. Conway, R. T. Curtis, S. P. Norton, R. A. Parker, and R. A. Wilson, An atlas of finite groups, Clarendon Press, Oxford, 1985.

6. W. Feit, The representation theory of finite groups, North-Holland, Amsterdam, 1982.

7. P. Fong, Solvable groups and modular representation theory, Trans. Amer. Math. Soc. 103 (1962), 484-494.

8. G. Hiss, A converse to the Fong-Swan-Isaacs theorem, J. Algebra 111 (1987), 279-290.

9. B. Huppert and N. Blackburn, Finite groups. III, Springer-Verlag, Berlin and New York, 1982.

10. M. Klemm, Charakterisierung der gruppen $\operatorname{PSL}\left(2, p^{f}\right)$ und $\operatorname{PSU}\left(3, p^{2 f}\right)$ durch ihrer charakterafel, J. Algebra 24 (1973), 127-153.

11. V. Landazuri and G. M. Seitz, On the minimal degrees of projective representations of the finite Chevalley groups, J. Algebra 32 (1974), 418-443.

12. M. Suzuki, On a class of doubly transitive groups, Ann. of Math. (2) 75 (1962), 104-145.

Department of Mathematics, University of Chicago, Chicago, Illinois 60637

Fachbereich Mathematik, Universität de Mainz, 6500 Mainz, Federal Republic of GERMANY 